\title{
On Properness and Protectiveness in Two-Person Multicriteria Games
}

\author{
M. Quant • P. Borm • G. Fiestras-Janeiro • \\ F. van Megen
}

Published online: 23 September 2008

(C) The Author(s) 2008. This article is published with open access at Springerlink.com

\begin{abstract}
This paper extends the concepts of proper equilibria, protective behavior and prudent behavior to multicriteria games. Three types of proper equilibria based on different types of domination are introduced. It is shown that protective behavior coincides with prudent behavior. Possible relations and existence are analyzed.
\end{abstract}

Keywords Multicriteria games · Proper behavior · Protective behavior · Prudent behavior

\section{Introduction}

Agents involved in competitive, interactive decision-making processes usually take into account various objectives. In these circumstances, the decision process can be modelled as a multicriteria game.

Multicriteria games have been introduced in [1], where repeated zero-sum games with vector payoffs are analyzed and an analog of the minimax theorem is derived. The notion of equilibrium, which in this setting is referred to as Pareto equilibrium,

Communicated by F. Zirilli.

M.G. Fiestras-Janeiro acknowlegdes the hospitality of the Department of Econometrics at Tilburg University and the financial support from Spanish Ministerio de Ciencia y Tecnología and FEDER through Project SEJ2005-07637-C02-02 as well as from the Xunta de Galicia, Project PGIDIT06PXIC207038PN.

M. Quant $(\varangle) \cdot$ P. Borm · F. van Megen

Department of Econometrics \& OR and CentER, Tilburg University, P.O. Box 90153, 5000 LE, Tilburg, Netherlands e-mail: Quant@uvt.nl

G. Fiestras-Janeiro

Departemento de Estatística e Investigación Operativa, Universidade de Vigo, Campus Lagoas-Marcosende, 36310 Vigo, Spain 
has been introduced in [2]. Pareto equilibria can be characterized as Nash equilibria (see [3]) of so called tradeoff (unicriterion) games, where each agent's payoff is obtained by a scalarization of his payoff in each objective with nonnegative weights. In [4], this result is extended to strong Pareto equilibria and positive weights. In [5], solutions for multicriteria games have been obtained by means of analyzing proper efficient solutions of corresponding vector maximization problems. In the case of two-person multicriteria games, the set of solutions equals the set of Pareto equilibria as a consequence of the Geoffrion representation theorem (see [6]).

As far as we know, little attention has been devoted to refining the set of Pareto equilibria for multicriteria games based on ideas similar to the refinements of Nash equilibria for unicriterion games. Based on the ideas behind the concept of perfect Nash equilibrium as considered in [7,8] proposed the concept of perfect equilibrium as a refinement of the set of Pareto equilibria. Explicitly considering the possibility to order the strategies of each player by means of levels of best reply sets, [8] also hinted at an extension of the concept of proper equilibria (see [9]) to a multicriteria setting.

In this paper, we analyze three extensions of the notion of proper equilibria based on different types of domination to two-person multicriteria games. These types are based on pure domination (or vector domination) and two forms of level domination, in which the strategies are divided in different levels of best replies. It is seen that the set of proper equilibria based on pure domination contains the set of proper equilibria of tradeoff games as a subset. Furthermore, it contains the sets of proper equilibria based on level domination. The extensions are illustrated by means of an example, clarifying the differences between the three types of domination.

Furthermore, we study an extension of the protective and prudent behavior to twoperson multicriteria games. Basically, protective and prudent strategies of a player deal with the (lexicographic) maximization of his worst possible payoff with respect to all the pure strategies of the other player. The two concepts differ in the case of inconclusiveness. A protective strategy then also searches for minimality in terms of inclusion of the sets of pure strategies which give rise to this worst payoff, whereas a prudent strategy aims of minimizing the cardinality of the set of pure strategies causing this worst payoff. We show that the protective behavior coincides with the prudent behavior and prove existence. Two examples illustrate some special aspects of protective behavior in a zero-sum like multicriteria environment and in particular highlight the difference with proper equilibria. One might have expected such a relationship on the basis of [10], which studies protective and prudent behavior in unicriterion games. It proves that, for matrix games, the set of protective equilibria coincides with the set of proper equilibria.

This paper is organized as follows. Section 2 contains the necessary definitions on two-person multicriteria games, Pareto equilibria, and efficient pure best reply sets. It also includes the correspondence between Pareto equilibria and Nash equilibria of the tradeoff games, and the characterization of the carriers of best reply strategies as a subset of efficient best reply sets. In Sect. 3, we introduce the notions of pure domination proper equilibrium, level domination proper equilibrium, and level proper equilibrium. An example illustrates these concepts and clarifies the differences among them. We prove the existence of pure domination proper equilibrium for any multicriteria game. In Sect. 4, we propose an extension of protective and 
prudent behavior to multicriteria games. We show that protective behavior coincides with prudent behavior, and prove existence. Two examples illustrate some aspects of protective behavior in a zero-sum like environment.

\section{Preliminaries: Multicriteria Games}

We consider mixed extensions of two-person finite strategic multicriteria games, in which player one can choose between $m$ and player two between $n$ pure strategies. The sets $\left\{e_{1}, \ldots, e_{m}\right\}$ and $\left\{f_{1}, \ldots, f_{n}\right\}$ contain the pure strategies of player one and two respectively. Player one and two take into account $r$ and $s$ criteria respectively. Let $A=\left(A_{1}, \ldots, A_{r}\right)$ and $B=\left(B_{1}, \ldots, B_{s}\right)$ be two vectors of real valued $m \times n$ matrices in which the rows correspond to pure strategies $e_{1}, \ldots, e_{m}$ and the columns to pure strategies $f_{1}, \ldots, f_{n}$. The matrix $A_{t}\left(B_{t}\right)$ can be interpreted as the payoff matrix of player one (two) with respect to criterium $t$. A two-person multicriteria game can then be described by a tuple $\Gamma=\left\langle\Delta_{m}, \Delta_{n}, u_{1}, u_{2}\right\rangle$. Here $\Delta_{m}$, a shorthand for $\Delta\left\{e_{1}, \ldots, e_{m}\right\}$, denotes the set of all mixed strategies of player one and $\Delta_{n}$ $\left(=\Delta\left\{f_{1}, \ldots, f_{n}\right\}\right)$ is the set of all mixed strategies of player two. The (relative) interior of e.g. $\Delta_{m}$ is denoted by $\AA_{m}$ : it represents the set of all completely mixed strategies. The functions $u_{1}$ and $u_{2}$ are the (vector) payoff functions of player one and two. For all $(p, q) \in \Delta_{m} \times \Delta_{n}$

$$
u_{1}(p, q)=\left(p A_{1} q, \ldots, p A_{r} q\right), \quad u_{2}(p, q)=\left(p B_{1} q, \ldots, p B_{s} q\right),
$$

or, in short notation,

$$
u_{1}(p, q)=p A q, \quad u_{2}(p, q)=p B q .
$$

We will usually describe a two-person multicriteria game by the tuple $\Gamma=$ $\left\langle\Delta_{m}, \Delta_{n}, A, B\right\rangle$. The set of all $r \times s$ multicriteria games of this type is denoted by $M G(r \times s, m \times n)$.

The (Pareto) equilibria of multicriteria games can be described by the notion of best reply functions. Take strategies $p \in \Delta_{m}$ and $q \in \Delta_{n}$. Then $p$ is a best reply to $q$ $\left(p \in B_{1}(\Gamma, q)\right)$ if there is no strategy $\bar{p} \in \Delta_{m}$ such that $p A q<\bar{p} A q$ (i.e. $(p A q)_{t}<$ $(\bar{p} A q)_{t}$ for all $\left.t \in\{1, \ldots r\}\right)$. Similarly $q$ is a best reply to $p\left(q \in B_{2}(\Gamma, p)\right)$ if there is no strategy $\bar{q} \in \Delta_{n}$ such that $p B q<p B \bar{q}$. The strategy combination $(p, q)$ is an equilibrium of $\Gamma$ if $p$ is a best reply to $q$ and $q$ is a best reply to $p$. The set of all equilibria of $\Gamma$ is denoted by $E(\Gamma)$.

There is a direct connection between multicriteria games and their corresponding tradeoff games in which the various criteria of each player are weighted.

Definition 2.1 Let $\Gamma=\left\langle\Delta_{m}, \Delta_{n}, A, B\right\rangle \in M G(r \times s, m \times n)$ be a two-person multicriteria game and let $\lambda_{1} \in \Delta_{r}, \lambda_{2} \in \Delta_{S}$ be tradeoff (or weight) vectors for player one and two respectively. Denote $\lambda=\left(\lambda_{1}, \lambda_{2}\right)$. The unicriterion game $\Gamma(\lambda)$ is defined as the bimatrix game with mixed strategy spaces $\Delta_{m}$ and $\Delta_{n}$ and $m \times n$ payoff matrices $A\left(\lambda_{1}\right), B\left(\lambda_{2}\right)$ given by

$$
A\left(\lambda_{1}\right)_{i j}=\sum_{t=1}^{r}\left(\lambda_{1}\right)_{t}\left(A_{t}\right)_{i j} \quad \text { and } \quad B\left(\lambda_{2}\right)_{i j}=\sum_{t=1}^{s}\left(\lambda_{2}\right)_{t}\left(B_{t}\right)_{i j}
$$


The set of all Nash equilibria of the bimatrix game $\Gamma(\lambda)$ is denoted by $\mathrm{NE}(\Gamma(\lambda))$.

The following theorem states that each equilibrium of a multicriteria game $\Gamma$ can be found as a Nash equilibrium of an unicriterion game, derived from $\Gamma$ by using a suitable tradeoff vector.

Theorem 2.1 (Shapley, [2]) Let $\Gamma=\left\langle\Delta_{m}, \Delta_{n}, A, B\right\rangle$ be a multicriteria game. Then,

$$
\left.E(\Gamma)=\left\{(p, q) \in \Delta_{m} \times \Delta_{n} \mid(p, q) \in N E(\Gamma(\lambda)) \text { for some } \lambda \in \Delta_{r} \times \Delta_{s}\right)\right\} .
$$

Reference [8] provides a characterization of (Pareto) equilibria points in terms of carriers and efficient best reply sets. Let $(p, q) \in \Delta_{m} \times \Delta_{n}$. The carrier of $p$ with respect to $\Gamma=\left\langle\Delta_{m}, \Delta_{n}, A, B\right\rangle$ is defined as $C(\Gamma, p):=\left\{i \in\{1, \ldots, m\} \mid p_{i}>0\right\}$. $I \subseteq\{1, \ldots, m\}$ is efficient for player one with respect to $q$ in $\Gamma$ if for all strategies $p \in$ $\Delta_{m}$ with $C(\Gamma, p) \subseteq I$ it holds that $p A q$ is undominated in the polytope $P_{1}(\Gamma, q):=$ $\operatorname{conv}\left\{e_{i} A q \mid i \in\{1, \ldots m\}\right\} \subset \mathbb{R}^{r}$ of all possible payoff vectors with respect to $q$. Note that if $I \subseteq\{1, \ldots, m\}$ is efficient, then each subset $K \subseteq I$ is efficient too. $I \subseteq$ $\{1, \ldots, m\}$ is an efficient pure best reply set for player one with respect to $q$ in $\Gamma$ if $I$ is efficient with respect to $q$ in $\Gamma$ and there does not exist an efficient set $K \subseteq\{1, \ldots, m\}$ such that $I \subseteq K$ and $I \neq K$. The set of all efficient pure best reply sets of player one with respect to $q$ in $\Gamma$ is denoted by $\mathcal{E}_{1}(\Gamma, q)$. In the same way one can define efficient sets and efficient pure best reply sets for player two. The following theorem shows the connection between best replies and efficient pure best reply sets.

Theorem 2.2 (Borm et al. [8]) Let $\Gamma=\left\langle\Delta_{m}, \Delta_{n}, A, B\right\rangle$ be a multicriteria game and $(p, q) \in \Delta_{m} \times \Delta_{n}$. Then, $p \in B_{1}(\Gamma, q)$ if and only if $C(\Gamma, p) \subseteq I$ for some $I \in \mathcal{E}_{1}(\Gamma, q)$ and $q \in B_{2}(\Gamma, p)$ if and only if $C(\Gamma, q) \subseteq J$ for some $J \in \mathcal{E}_{2}(\Gamma, p)$.

\section{Proper Equilibria}

In this section, we introduce three types of proper equilibria of multicriteria games.

Reference [8] introduces perfect equilibria of multicriteria games, generalizing the notion of perfect Nash equilibria introduced by [7]. Take $\varepsilon>0$. A strategy combination $(p, q) \in \stackrel{\circ}{\Delta}_{m} \times \stackrel{\circ}{\Delta}_{n}$ is called an $\varepsilon$-perfect pair if there exists an $I \in \mathcal{E}_{1}(\Gamma, q)$ and an $J \in \mathcal{E}_{2}(\Gamma, p)$ such that $p_{i} \leq \varepsilon$, for all $i \notin I$ and $q_{j} \leq \varepsilon$ for all $j \notin J$.

Definition 3.1 Let $\Gamma=\left\langle\Delta_{m}, \Delta_{n}, A, B\right\rangle$ be a two-person multicriteria game. A strategy combination $(p, q)$ is a perfect equilibrium of $\Gamma$ if there exists a sequence $\left\{\varepsilon_{t}\right\}_{t=1}^{\infty} \subset(0, \infty)$ converging to zero and a sequence $\left\{\left(p^{t}, q^{t}\right)\right\}_{t=1}^{\infty} \subset \stackrel{\circ}{\Delta}_{m} \times \stackrel{\circ}{n}_{n}$ converging to $(p, q)$, such that $\left(p^{t}, q^{t}\right)$ is an $\varepsilon_{t}$-perfect pair for all $t$.

Another refinement of Nash equilibria are the proper equilibria, introduced in [9]. Let $\Gamma=\left(\Delta_{m}, \Delta_{n}, A, B\right)$ be an $m \times n$ bimatrix game, i.e. $\Gamma \in M G(1 \times 1, m \times n)$. Take $\varepsilon \in(0,1)$. Then, a strategy combination $(p, q) \in \stackrel{\circ}{\Delta}_{m} \times \stackrel{\circ}{\Delta}_{n}$ is an $\varepsilon$-proper pair if, for all $i, k \in\{1, \ldots, m\}, j, l \in\{1, \ldots, n\}$, we have

$$
e_{i} A q<e_{k} A q \Rightarrow p_{i} \leq \varepsilon p_{k},
$$




$$
p B f_{j}<p B f_{l} \Rightarrow q_{j} \leq \varepsilon q_{l} .
$$

A strategy combination $(p, q) \in \Delta_{m} \times \Delta_{n}$ is a proper equilibrium of $\Gamma$ if there exist a sequence $\left\{\varepsilon_{t}\right\}_{t=1}^{\infty}$ of positive numbers converging to zero and a sequence $\left\{\left(p^{t}, q^{t}\right)\right\}_{t=1}^{\infty}$ of completely mixed strategy combinations converging to $(p, q)$ such that $\left(p^{t}, q^{t}\right)$ is an $\varepsilon_{t}$-proper pair for all $t \in \mathbb{N}$. The set of all proper equilibria of $\Gamma$ is denoted by $P R(\Gamma)$.

The idea behind proper equilibria is that costly mistakes occur with relatively smaller probabilities than less costly mistakes, whereas in perfect equilibria mistakes are not compared at all. Note that in unicriterion games any two pure strategies can be mutually compared and as a consequence the set of pure strategies can be partitioned into well-defined levels of quality, given a strategy of the opponent. In a multicriteria environment we do not have this completeness and there are several options on how to deal with this. We introduce three types of proper equilibria in multicriteria games each based on another way of comparing pure strategies. To do so, we first introduce some terminology.

Let

$$
\Gamma=\left\langle\Delta_{m}, \Delta_{n}, A, B\right\rangle \in M G(r \times s, m \times n)
$$

and take $q \in \Delta_{n}$. The first level of best replies of player one against $q$ is the set of all pure strategies contained in some efficient pure best reply sets with respect to $q$. The $t$ 'th level of best replies is constructed by considering the best replies with respect to $q$ taking into account all strategies that are not in the first $t-1$ levels. Formally this boils down to the following definition:

$$
\begin{aligned}
& M^{1}(q):=\{1, \ldots, m\}, \quad m^{1}(q):=m, \\
& \Gamma_{1}^{1}(q):=\Gamma=\left\langle\Delta_{m}, \Delta_{n}, A, B\right\rangle, \\
& \mathcal{E}_{1}^{1}(\Gamma, q):=\mathcal{E}_{1}\left(\Gamma_{1}^{1}(q), q\right), \\
& L_{1}^{1}(q):=\left\{i \in M^{1}(q) \mid i \in I \text { for some } I \in \mathcal{E}_{1}^{1}(\Gamma, q)\right\},
\end{aligned}
$$

recursively, for each $t \in \mathbb{N}, t \geq 2$ :

$$
\begin{aligned}
& M^{t}(q):=M^{t-1}(q) \backslash L_{1}^{t-1}(q), \quad m^{t}(q):=\left|M^{t}(q)\right|, \\
& \Gamma_{1}^{t}(q):=\left\langle\Delta_{m^{t}(q)}, \Delta_{n}, A^{t}, B^{t}\right\rangle, \\
& \mathcal{E}_{1}^{t}(\Gamma, q):=\mathcal{E}_{1}\left(\Gamma_{1}^{t}(q), q\right), \\
& L_{1}^{t}(q):=\left\{i \in M^{t}(q) \mid i \in I \text { for some } I \in \mathcal{E}_{1}^{t}(\Gamma, q)\right\} .
\end{aligned}
$$

Here, $\Delta_{m^{t}(q)}=\Delta\left(\left\{e_{i}\right\}_{i \in M^{t}(q)}\right)$ is the restricted mixed strategy space and $A^{t}$ and $B^{t}$ are the corresponding vectors of $m^{t}(q) \times n$ submatrices of the vectors of matrices $A$ and $B$ corresponding to the rows in $M^{t}(q)$. Note that there is an unique $z \in \mathbb{N}$ such that $M^{z-1}(q) \neq \emptyset$ and $M^{z}(q)=\emptyset$. In the same way, one can define $N^{t}(p), n^{t}(p)$, $\Gamma_{2}^{t}(p), \mathcal{E}_{2}^{t}(\Gamma, p)$ and $L_{2}^{t}(p)$ for $p \in \Delta_{m}$.

Let $\varepsilon \in(0,1)$ and $\Gamma=\left\langle\Delta_{m}, \Delta_{n}, A, B\right\rangle$ be a multicriteria game. The completely mixed strategy combination $(p, q) \in \stackrel{\circ}{\Delta}_{m} \times \stackrel{\circ}{\Delta}_{n}$ is

- a pure domination $\varepsilon$-proper pair of $\Gamma$ if, for all $i, k \in\{1, \ldots, m\}$ such that $e_{i} A q<$ $e_{k} A q$, it holds that $p_{i} \leq \varepsilon p_{k}$ and if, for all $j, l \in\{1, \ldots, n\}$ such that $p B f_{j}<p B f_{l}$, it holds that $q_{j} \leq \varepsilon q_{l}$. 
- a level domination $\varepsilon$-proper pair if for all $i \in L_{1}^{t}(q)$ : if there is a $\bar{p} \in \Delta_{m}$ and $I \in \mathcal{E}_{1}^{t-1}(\Gamma, q)$ with $C(\bar{p}) \subseteq I$ such that $e_{i} A q \leq \bar{p} A q$, then $p_{i} \leq \varepsilon p_{k}$ for all $k \in I$ and if for all $j \in L_{2}^{t}(p)$ : if there is a $\bar{q} \in \Delta_{n}$ and $J \in \mathcal{E}_{2}^{t-1}(\Gamma, p)$ with $C(\bar{q}) \subseteq J$ such that $p B f_{j} \leq p B \bar{q}$, then $q_{j} \leq \varepsilon q_{l}$ for all $l \in J$.

- a level $\varepsilon$-proper pair if for all $i, k \in\{1, \ldots, m\}$ : if $i \in L_{1}^{t}(q)$ and $k \in L_{1}^{t-1}(q)$, then $p_{i} \leq \varepsilon p_{k}$ and if for all $j, l \in\{1, \ldots, n\}:$ if $j \in L_{2}^{t}(p)$ and $l \in L_{2}^{t-1}(p)$, then $q_{j} \leq \varepsilon q_{l}$.

We now introduce three types of proper equilibria in multicriteria games. The difference between the three types of proper equilibria are due to the different notions of domination between pure strategies used. The first type of proper equilibria is based on standard vector domination in all coordinates (criteria), this leads to pure domination proper equilibria. In the second type of domination a strategy $e_{i}$ dominates $e_{k}$, if $e_{i}$ is contained in an efficient pure best reply set of a lower level such that there exist a mixed strategy (with carrier within this pure best reply set) such that $e_{k}$ is dominated in all coordinates by this mixed strategy. This second type of proper equilibria is called level domination proper equilibria. Finally, in the third type of domination a strategy of a higher level is dominated by all strategies of lower levels. This leads to level proper equilibria. Note that in unicriterion games all three concepts of domination coincide. The idea of level proper equilibria is shortly mentioned in [8].

Definition 3.2 Let $\Gamma=\left\langle\Delta_{m}, \Delta_{n}, A, B\right\rangle$ be a multicriteria game. The strategy combination $(p, q) \in \Delta_{m} \times \Delta_{n}$ is a pure domination proper equilibrium of $\Gamma$ if $(p, q)$ is a perfect equilibrium of $\Gamma$ and there exists a sequence $\left\{\varepsilon_{t}\right\}_{t=1}^{\infty} \subset(0,1)$ converging to zero and a sequence $\left\{\left(p^{t}, q^{t}\right)\right\}_{t=1}^{\infty} \subset \stackrel{\circ}{\Delta}_{m} \times \stackrel{\circ}{\Delta}_{n}$ converging to $(p, q)$, such that $\left(p^{t}, q^{t}\right)$ is a dominated $\varepsilon_{t}$-proper pair for all $t$. Similarly the strategy combination $(p, q) \in \Delta_{m} \times \Delta_{n}$ is a level domination proper (level proper) equilibrium of $\Gamma$ if $(p, q)$ is a perfect equilibrium of $\Gamma$ and there exists a sequence $\left\{\varepsilon_{t}\right\}_{t=1}^{\infty} \subset(0,1)$ converging to zero and a sequence $\left\{\left(p^{t}, q^{t}\right)\right\}_{t=1}^{\infty} \in \stackrel{\circ}{\Delta}_{m} \times \stackrel{\circ}{\Delta}_{n}$ converging to $(p, q)$, such that $\left(p^{t}, q^{t}\right)$ is a level domination (level proper) $\varepsilon_{t}$-proper pair for all $t$.

The set of pure domination proper equilibria of a multicriteria game $\Gamma$ is denoted by $P D P(\Gamma)$. The sets of level domination proper equilibria and level proper equilibria are denoted by $L D P(\Gamma)$ and $L P(\Gamma)$ respectively.

Proposition 3.1 Let $\Gamma$ be a multicriteria game. Then, $L P(\Gamma) \subseteq L D P(\Gamma) \subseteq$ $P D P(\Gamma)$.

Proof It suffices to note that each level $\varepsilon$-proper pair is a level domination $\varepsilon$-proper pair and each level domination $\varepsilon$-proper pair is a pure domination $\varepsilon$-proper pair.

In the following theorem it is shown that the set of pure domination proper equilibria contains the set of proper equilibria of all tradeoff games.

Theorem 3.1 Let $\Gamma=\left\langle\Delta_{m}, \Delta_{n}, A, B\right\rangle$ be a multicriteria game. Then, we have that

$$
\left\{(p, q) \in \Delta_{m} \times \Delta_{n} \mid \exists \lambda \in \Delta_{r} \times \Delta_{s}:(p, q) \in P R(\Gamma(\lambda))\right\} \subseteq P D P(\Gamma) .
$$


Proof Let

$$
\lambda=\left(\lambda_{1}, \lambda_{2}\right) \in \Delta_{r} \times \Delta_{S} \quad \text { and } \quad(p, q) \in P R(\Gamma(\lambda)) .
$$

We first show that $(p, q) \in P E(\Gamma)$. This follows immediately from Theorem 5.3 in [8] and the fact that in unicriterion games the set of proper equilibria is contained in the set of perfect equilibria.

Furthermore, since $(p, q)$ is a proper equilibrium in the unicriterion game $\Gamma(\lambda)$, there exist sequences $\left\{\varepsilon_{t}\right\}_{t=1}^{\infty} \subset(0,1)$ and $\left\{\left(p^{t}, q^{t}\right)\right\}_{t=1}^{\infty} \subset \stackrel{\circ}{\Delta}_{m} \times \stackrel{\circ}{\Delta}_{n}$ such that $\lim _{t \rightarrow \infty} \varepsilon_{t}=0$ and $\lim _{k \rightarrow \infty}\left(p^{t}, q^{t}\right)=(p, q)$ and $\left(p^{t}, q^{t}\right)$ is $\varepsilon_{t}$-proper in $\Gamma(\lambda)$ for all $t \in \mathbb{N}$. Let $t \in \mathbb{N}$ and let $i, k \in\{1, \ldots, m\}$ be such that

$$
e_{i} A q^{t}<e_{k} A q^{t}
$$

Clearly, we are finished if we can show that $p_{i}^{t} \leq \varepsilon_{t} p_{k}^{t}$.

Since $\lambda_{1} \geq 0$ it is true that

$$
\begin{aligned}
e_{i} A(\lambda) q^{t} & =\sum_{d=1}^{r}\left(\lambda_{1}\right)_{d} e_{i} A_{d} q^{t} \\
& <\sum_{d=1}^{r}\left(\lambda_{1}\right)_{d} e_{k} A_{d} q^{t} \\
& =e_{k} A(\lambda) q^{t} .
\end{aligned}
$$

From the fact that $\left(p^{t}, q^{t}\right)$ is $\varepsilon_{t}$-proper in $\Gamma(\lambda)$, it then follows that

$$
p_{i}^{t} \leq \varepsilon_{t} p_{k}^{t}
$$

Now the existence of pure domination proper equilibria follows easily from the existence of proper equilibria in unicriterion games as proved in [9]:

Corollary 3.1 For every multicriteria game, the set of pure domination proper equilibria is nonempty. ${ }^{1}$

The following example illustrates the differences between the three concepts of properness.

Example 3.1 Consider a $(2 \times 1,5 \times 4)$ multicriteria game $\Gamma$ with payoff matrix $A$ and $B$ given by

$$
A=\left(\begin{array}{llll}
(2,3) & (2,3) & (2,3) & (2,3) \\
(0,3) & (0,3) & (0,3) & (0,3) \\
(6,0) & (6,0) & (6,0) & (6,0) \\
(3,2) & (3,2) & (3,2) & (3,2) \\
(2,1) & (2,1) & (2,1) & (2,1)
\end{array}\right)
$$

\footnotetext{
${ }^{1}$ We conjecture that the sets of level domination proper and level proper equilibria are nonempty as well.
} 
Fig. 1 Possible payoff vectors of player one (independent of player 2's strategy choice)

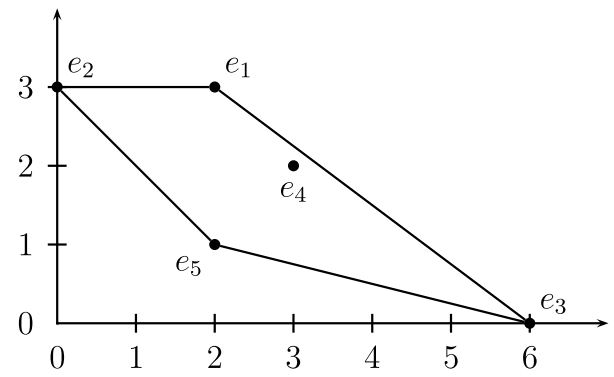

and

$$
B=\left(\begin{array}{llll}
1 & 1 & 1 & 1 \\
2 & 0 & 1 & 1 \\
1 & 1 & 0 & 1 \\
0 & 2 & 2 & 0 \\
1 & 1 & 1 & 2
\end{array}\right),
$$

respectively. Note that the vector payoff of player one is independent of the strategy chosen by player two. The set of possible payoff vectors of player one, $\operatorname{conv}\left\{e_{i} A q \mid\right.$ $i \in\{1, \ldots, 5\}\}$, does not depend on $q$ and is drawn in Fig. 1. From this figure it is immediately clear that for all $q \in \Delta_{4}$ the set of first level efficient pure best reply sets is given by $\mathcal{E}_{1}^{1}(\Gamma, q)=\{\{1,2\},\{1,3\}\}$, second, that $\mathcal{E}_{1}^{2}(\Gamma, q)=\{\{4\}\}$ and $\mathcal{E}_{1}^{3}(\Gamma, q)=$ $\{\{5\}\}$. It can be verified easily that the set of perfect equilibria equals the set of Nash equilibria

$$
\begin{aligned}
E(\Gamma)= & \operatorname{conv}\left\{e_{1}, e_{3}\right\} \times \operatorname{conv}\left\{f_{1}, f_{2}, f_{4}\right\} \cup \operatorname{conv}\left\{e_{1}, e_{2}\right\} \times\left\{f_{1}\right\} \\
& \cup\left\{e_{1}\right\} \times \operatorname{conv}\left\{f_{1}, f_{2}, f_{3}, f_{4}\right\} \\
= & P E(\Gamma) .
\end{aligned}
$$

To find all pure domination proper equilibria, note that the strategy $e_{5}$ of player one is purely dominated by $e_{4}$. Let $\varepsilon>0$ and $(p, q)$ a pure domination $\varepsilon$-proper pair. Then it holds that $f_{4}$ is dominated by at least one strategy: $p B f_{1}>p B f_{4}$ or $p B f_{2}>p B f_{4}$, (or both), from which it follows that for a pure domination proper equilibrium $(p, q)$ it holds that $q_{4}=0$. By choosing suitable sequences, one can show that all remaining equilibria are pure domination proper. Therefore, the set of all pure domination proper equilibria equals

$$
\begin{aligned}
P D P(\Gamma)= & \operatorname{conv}\left\{e_{1}, e_{3}\right\} \times \operatorname{conv}\left\{f_{1}, f_{2}\right\} \\
& \cup \operatorname{conv}\left\{e_{1}, e_{2}\right\} \times\left\{f_{1}\right\} \\
& \cup\left\{e_{1}\right\} \times \operatorname{conv}\left\{f_{1}, f_{2}, f_{3}\right\} .
\end{aligned}
$$

To find all level domination proper equilibria, note that for all $q \in \Delta_{4}, e_{4}$ is dominated by $\bar{p}=\frac{7}{10} e_{1}+\frac{3}{10} e_{3}$ and the carrier of $\bar{p}$ equals $\{1,3\}$. Hence, if $(p, q)$ is a level domination $\varepsilon$-proper pair, then it holds that $p_{5} \leq \varepsilon p_{4}, p_{4} \leq \varepsilon p_{1}, p_{4} \leq \varepsilon p_{3}$. It follows 
that $p B f_{3} \leq p B f_{1}$ for small $\varepsilon$. If $p_{2}=p_{4}$, both $f_{1}$ and $f_{2}$ are best replies. The set of level domination proper equilibria becomes

$$
\begin{aligned}
\operatorname{LDP}(\Gamma)= & \operatorname{conv}\left\{e_{1}, e_{3}\right\} \times \operatorname{conv}\left\{f_{1}, f_{2}\right\} \\
& \cup \operatorname{conv}\left\{e_{1}, e_{2}\right\} \times\left\{f_{1}\right\} \\
& \cup\left\{e_{1}\right\} \times \operatorname{conv}\left\{f_{1}, f_{2}\right\} .
\end{aligned}
$$

Suppose that $(p, q)$ is a level $\varepsilon$-proper pair. Then, the following inequalities are true $p_{5} \leq \varepsilon p_{4}$ and $p_{4} \leq \varepsilon p_{k}$, with $k \in\{1,2,3\}$. Consequently,

$$
p B f_{2}<p B f_{1}, \quad p B f_{3}<p B f_{1}, \quad p B f_{4}<p B f_{1}
$$

and the set of level proper equilibria is given by

$$
L P(\Gamma)=\operatorname{conv}\left\{e_{1}, e_{3}\right\} \times\left\{f_{1}\right\} \cup \operatorname{conv}\left\{e_{1}, e_{2}\right\} \times\left\{f_{1}\right\} .
$$

\section{Protective Behavior}

Protective and prudent strategies for mixed extensions of finite games are introduced in [10]. Generally speaking a protective strategy of a player maximizes his worst possible payoff with respect to all pure strategy combinations of the other players. In case of inconclusiveness it also searches for minimality in terms of inclusion of the sets of pure strategy combinations which give rise to this worst payoff. A prudent strategy also maximizes the worst possible payoff with respect to all pure strategy combinations of the other players, but on the secondary level it aims to minimize the cardinality of the sets of pure strategy combinations causing this worst payoff.

Reference [10] shows that the notions of prudent and protective are in fact equivalent and prove existence. Moreover, it turns out that for matrix games the set of protective strategy combinations coincides with the set of proper equilibria.

In this section, we introduce protective and prudent strategies for our setting of (two-person) multicriteria games. The equivalence of protective and prudent strategies is shown and existence is proved. By means of an example we show that even for zero-sum like games protective strategy combinations need not be Pareto equilibria.

Let $\theta: \mathbb{R}^{u} \rightarrow \mathbb{R}^{u}$ be the map that assigns to any $z \in \mathbb{R}^{u}$ the vector $\theta(z)$ which orders the coordinates of $z$ in a weakly increasing order.

Let $\Gamma=\left\langle\Delta_{m}, \Delta_{n}, A, B\right\rangle$ be a multicriteria game. Let $\bar{p} \in \Delta_{m}$. The pure strategies of player 2 are compared on the basis of a worst case scenario taking all criteria into account with equal importance. Hence the payoffs corresponding to the several criteria are ordered in a weakly increasing order and then compared by using the lexicographic ordering $\left(\preceq_{L}\right)$. Recursively, we define the vector $a^{t}(\bar{p}) \in \mathbb{R}^{r}$ and the sets $Z^{t}(\bar{p})$ and $J^{t}(\bar{p})$ by

(i) for $t=1$,

$$
\begin{aligned}
Z^{1}(\bar{p})= & \left\{z \in \mathbb{R}^{r} \mid \text { there is a } k \in\{1, \ldots, n\} \text { such that } \bar{p} A f_{k}=z\right. \\
& \text { and such that } \left.\theta\left(\bar{p} A f_{k}\right) \preceq_{L} \theta\left(\bar{p} A f_{j}\right) \text { for all } j \in\{1, \ldots, n\}\right\},
\end{aligned}
$$




$$
\begin{aligned}
& a^{1}(\bar{p})={ }^{2} \theta(z), \quad \text { for all } z \in Z^{1}(\bar{p}), \\
& J^{1}(\bar{p})=\left\{j \in\{1, \ldots, n\} \mid \bar{p} A f_{j} \in Z^{1}(\bar{p})\right\} .
\end{aligned}
$$

(ii) for $t>1$,

$$
\begin{aligned}
& Z^{t}(\bar{p})=\left\{z \in \mathbb{R}^{r} \mid \text { there is a } k \in\{1, \ldots, n\} \backslash \bigcup_{l=1}^{t-1} J^{l}(\bar{p})\right. \text { such that } \\
& \bar{p} A f_{k}=z \text { and such that } \theta\left(\bar{p} A f_{k}\right){ }_{L} \theta\left(\bar{p} A f_{j}\right) \text { for all } \\
& \left.j \in\{1, \ldots, n\} \backslash \bigcup_{l=1}^{t-1} J^{l}(\bar{p})\right\}, \\
& a^{t}(\bar{p})=\theta(z), \quad \text { for all } z \in Z^{t}(\bar{p}), \\
& J^{t}(\bar{p})=\left\{j \in\{1, \ldots, n\} \mid \bar{p} A f_{j} \in Z^{t}(\bar{p})\right\} .
\end{aligned}
$$

If $Z^{t}(\bar{p})=\emptyset$, then $J^{t}(\bar{p})=\emptyset$ and we define $a^{t}(\bar{p})=(\infty, \ldots, \infty)$. Similarly one can define the vector $b^{t}(\bar{q}) \in \mathbb{R}^{s}$ and the sets $Z^{t}(\bar{q})$ and $I^{t}(\bar{q})$.

Definition 4.1 Let $\Gamma=\left\langle\Delta_{m}, \Delta_{n}, A, B\right\rangle$ be a multicriteria game. Let $\bar{p}, p \in \Delta_{m}$. We say that $\bar{p}$ protectively dominates $p$, in notation, $\bar{p} \succ_{\text {pro }} p$, if there exists an $l \in \mathbb{N}$ such that

(i) $a^{t}(\bar{p})=a^{t}(p)$ and $J^{t}(\bar{p})=J^{t}(p)$ for all $t \in \mathbb{N}, t<l$,

(ii) $a^{l}(\bar{p}) \succ_{L} a^{l}(p)$ or, both $a^{l}(\bar{p})=a^{l}(p)$ and $J^{l}(\bar{p}) \varsubsetneqq J^{l}(p)$.

A mixed strategy $p \in \Delta_{m}$ is called protective for player 1 in $\Gamma$ if there does not exist a mixed strategy $\bar{p} \in \Delta_{m}$ such that $\bar{p} \succ_{\text {pro }} p$. In a similar way, one can define protective strategies for player 2 .

Even though the protective dominance relation need not to be complete, the next lemma reveals that a protective strategy is dominant, up to payoff equivalence, with respect to the relation $\succ_{\text {pro }}$. Here, two strategies are payoff equivalent if they yield the same payoff with respect to all criteria and all pure strategies of the other player. For this we first need a technical lemma.

Lemma 4.1 Let $\Gamma=\left\langle\Delta_{m}, \Delta_{n}, A, B\right\rangle$ be a multicriteria game. Consider $\bar{p}, p \in \Delta_{m}$ and define $\hat{p}=\alpha \bar{p}+(1-\alpha) p$ for some $\alpha \in(0,1)$. Moreover, let $j \in\{1, \ldots, n\}$ be such that $\bar{p} A f_{j} \neq p A f_{j}$. Then,

$$
\text { either } \theta\left(\hat{p} A f_{j}\right) \succ_{L} \theta\left(\bar{p} A f_{j}\right) \quad \text { or } \quad \theta\left(\hat{p} A f_{j}\right) \succ_{L} \theta\left(p A f_{j}\right) .
$$

Proof Let $d \in\{1, \ldots, r\}$. Then,

$$
\left(\hat{p} A f_{j}\right)_{d}=\alpha\left(\bar{p} A f_{j}\right)_{d}+(1-\alpha)\left(p A f_{j}\right)_{d}
$$

\footnotetext{
${ }^{2}$ Note that this vector is well-defined since the lexicographic minimum within the ordered sets is unique.
} 
and hence,

$$
\begin{aligned}
& \left(\hat{p} A f_{j}\right)_{d}=\left(\bar{p} A f_{j}\right)_{d}=\left(p A f_{j}\right)_{d}, \quad \text { if }\left(\bar{p} A f_{j}\right)_{d}=\left(p A f_{j}\right)_{d}, \\
& \left(\hat{p} A f_{j}\right)_{d}>\min \left\{\left(\bar{p} A f_{j}\right)_{d},\left(p A f_{j}\right)_{d}\right\}, \quad \text { if }\left(\bar{p} A f_{j}\right)_{d} \neq\left(p A f_{j}\right)_{d} .
\end{aligned}
$$

Let $\bar{\sigma}$ be an ordering of the criteria such that $\left(\bar{p} A f_{j}\right)_{\bar{\sigma}(u)}=\left(\theta\left(\bar{p} A f_{j}\right)\right)_{u}$ for all $u \in\{1, \ldots, r\}$ and $\bar{\sigma}(u)<\bar{\sigma}(v)$ whenever $\left(\theta\left(\bar{p} A f_{j}\right)\right)_{u}=\left(\theta\left(\bar{p} A f_{j}\right)\right)_{v}$ and $u<v$. In a similar way one can define $\sigma$ which gives an ordering of the criteria based on $\theta\left(p A f_{j}\right)$.

If $\bar{\sigma}$ and $\sigma$ are equal, then $\theta\left(\hat{p} A f_{j}\right)=\alpha \theta\left(\bar{p} A f_{j}\right)+(1-\alpha) \theta\left(p A f_{j}\right)$. Since $\bar{p} A f_{j} \neq$ $p A f_{j}$, we can assume without loss of generality that $\left.\theta\left(\bar{p} A f_{j}\right)\right) \succ_{L} \theta\left(p A f_{j}\right)$. Define $v$ as the smallest number such that $\left(\theta\left(\bar{p} A f_{j}\right)\right)_{v} \neq\left(\theta\left(p A f_{j}\right)\right)_{v}$. Then (1) is valid for all criteria $\sigma(l)$ with $l<v$. Inequality (2) is true for $d=\sigma(v)$, implying that $\left(\hat{p} A f_{j}\right)_{\sigma(v)}>\left(p A f_{j}\right)_{\sigma(v)}$. For all $l>v:\left(\bar{p} A f_{j}\right)_{\sigma(l)} \geq\left(\bar{p} A f_{j}\right)_{\sigma(v)}>\left(p A f_{j}\right)_{\sigma(v)}$, together with $\left(p A f_{j}\right)_{\sigma(l)} \geq\left(p A f_{j}\right)_{\sigma(v)}$, this indicates that $\left(\hat{p} A f_{j}\right)_{\sigma(l)}>\left(p A f_{j}\right)_{\sigma(v)}$. It can be concluded that $\theta\left(\hat{p} A f_{j}\right) \succ_{L} \theta\left(p A f_{j}\right)$.

Assume that $\bar{\sigma}$ and $\sigma$ are not equal. Let $u$ be the smallest number such that $\bar{\sigma}(u) \neq$ $\sigma(u)$. There are two cases.

In the first case $\left(\theta\left(\bar{p} A f_{j}\right)\right)_{l}=\left(\theta\left(p A f_{j}\right)\right)_{l}$ for all $l \in\{1, l \ldots, u\}$ and hence (1) is valid for all $l \in\{1, \ldots, u-1\}$, implying that

$$
\left(\hat{p} A f_{j}\right)_{\sigma(l)}=\left(\bar{p} A f_{j}\right)_{\sigma(l)}=\left(p A f_{j}\right)_{\sigma(l)}
$$

for all $l \in\{1, \ldots, u-1\}$. Without loss of generality we assume that $\sigma(u)<\bar{\sigma}(u)$. It follows from the definition of $\sigma$ and $\bar{\sigma}$ that $\left(\bar{p} A f_{j}\right)_{\sigma(u)}>\left(p A f_{j}\right)_{\sigma(u)}$. Using inequality (2), we find that

$$
\left(\hat{p} A f_{j}\right)_{\sigma(u)}>\left(p A f_{j}\right)_{\sigma(u)} .
$$

For all $l>u$, it is true that $\left(\bar{p} A f_{j}\right)_{\sigma(l)} \geq\left(\theta\left(\bar{p} A f_{j}\right)\right)_{u}=\left(\theta\left(p A f_{j}\right)\right)_{u}$ and $\left(p A f_{j}\right)_{\sigma(l)} \geq$ $\left(\theta\left(p A f_{j}\right)\right)_{u}$. Hence,

$$
\left(\hat{p} A f_{j}\right)_{\sigma(l)} \geq\left(\theta\left(p A f_{j}\right)\right)_{u},
$$

and equality holds if and only if $\left(\bar{p} A f_{j}\right)_{\sigma(l)}=\left(\theta\left(p A f_{j}\right)\right)_{u}=\left(p A f_{j}\right)_{\sigma(l)}$. We conclude from (3), (4) and (5) that

$$
\theta\left(\hat{p} A f_{j}\right) \succ_{L} \theta\left(p A f_{j}\right)
$$

In the second case there exists an $l \leq u$ such that $\left.\left(\theta\left(\bar{p} A f_{j}\right)\right)_{l} \neq \theta\left(p A f_{j}\right)\right)_{l}$. Let $v$ be the smallest number such that $\left.\left(\theta\left(\bar{p} A f_{j}\right)\right)_{v} \neq \theta\left(p A f_{j}\right)\right)_{v}$. Assume without loss of generality that $\theta\left(\bar{p} A f_{j}\right) \succ_{L} \theta\left(p A f_{j}\right)$. Then for all $l<v:\left(\bar{p} A f_{j}\right)_{\sigma(l)}=\left(p A f_{j}\right)_{\sigma(l)}$ and hence (1) is true with $d=\sigma(l)$. For all $l \geq v:\left(\bar{p} A f_{j}\right)_{\sigma(l)} \geq\left(\theta\left(\bar{p} A f_{j}\right)\right)_{v}>$ $\left(\theta\left(p A f_{j}\right)\right)_{v}=\left(p A f_{j}\right)_{\sigma(v)}$. Hence, by inequality (2),

$$
\left(\theta\left(\hat{p} A f_{j}\right)\right)_{\sigma(l)}>\min \left\{\left(\bar{p} A f_{j}\right)_{\sigma(l)},\left(p A f_{j}\right)_{\sigma(l)}\right\}>\left(\theta\left(p A f_{j}\right)\right)_{v} .
$$

It follows that $\theta\left(\hat{p} A f_{j}\right) \succ_{L} \theta\left(p A f_{j}\right)$. 
Lemma 4.2 Let $\Gamma=\left\langle\Delta_{m}, \Delta_{n}, A, B\right\rangle$ be a multicriteria game. Let $\bar{p}$ be a protective strategy for player 1 and let $p \in \Delta_{m}$. Then, either $\bar{p}$ and $p$ are payoff equivalent for player 1 or $\bar{p} \succ_{\text {pro }} p$.

Proof Suppose that $\bar{p}$ and $p$ are not payoff equivalent and that $\bar{p}$ does not protectively dominate $p$. Let $\alpha \in(0,1)$ and define $\hat{p}:=\alpha \bar{p}+(1-\alpha) p$. It is shown below that the strategy $\hat{p}$ protectively dominates $\bar{p}$, yielding a contradiction.

Because of our assumptions, there exists an $l \in \mathbb{N}$ such that

$$
\begin{aligned}
& a^{t}(\bar{p})=a^{t}(p) \quad \text { and } \quad J^{t}(\bar{p})=J^{t}(p), \quad \text { for all } t \in \mathbb{N}, t<l, \\
& a^{l}(\bar{p})=a^{l}(p), \quad J^{l}(\bar{p}) \backslash J^{l}(p) \neq \emptyset \quad \text { and } \quad J^{l}(p) \backslash J^{l}(\bar{p}) \neq \emptyset .
\end{aligned}
$$

We first prove that for all $j \in J^{t}(\bar{p})$, with $t<l$ it holds that $\bar{p} A f_{j}=p A f_{j}$ and hence $\bar{p} A f_{j}=p A f_{j}=\hat{p} A f_{j}$. Suppose not: pick the smallest $t<l$ for which we can find $j \in J^{t}(\bar{p})=J^{t}(p)$ such that $\bar{p} A f_{j} \neq p A f_{j}$. Since $j \in J^{t}(p)=J^{t}(\bar{p})$, it holds that $\theta\left(p A f_{j}\right)=\theta\left(\bar{p} A f_{j}\right)$. It follows from Lemma 4.1 that $\theta\left(\hat{p} A f_{j}\right) \succ_{L}$ $\theta\left(\bar{p} A f_{j}\right)=\theta\left(p A f_{j}\right)$. Let $k \in\{1, \ldots, n\} \backslash \bigcup_{\ell=1}^{t} J^{\ell}(\bar{p})$. Then $\theta\left(\bar{p} A f_{k}\right) \succ_{L} a^{t}(\bar{p})$, and $\theta\left(p A f_{k}\right) \succ_{L} a^{t}(p)=a^{t}(\bar{p})$. Using Lemma 4.1 yields: $\theta\left(\hat{p} A f_{k}\right) \succ_{L} a^{t}(\bar{p})$. Clearly for all $u<t: a^{u}(\hat{p})=a^{u}(\bar{p})$, and $J^{u}(\hat{p})=J^{u}(\bar{p})=J^{u}(p)$, furthermore $a^{t}(\hat{p}) \succ_{L}$ $a^{t}(\bar{p})$ or $a^{t}(\hat{p})=a^{t}(\bar{p})$ and $J^{t}(\hat{p}) \subset J^{t}(\bar{p})$ (since $j \in J^{t}(\bar{p})$, but $j \notin J^{t}(\hat{p})$ ). We can conclude that $\hat{p} \succ_{\text {prot }} \bar{p}$, yielding a contradiction.

Secondly, it can be proved in the same way as above that for all $j \in J^{l}(\bar{p}) \cap J^{l}(p)$ it is true that $\bar{p} A f_{j}=p A f_{j}$.

There are now two cases: $J^{l}(\bar{p}) \cap J^{l}(p)=\emptyset$ and $J^{l}(\bar{p}) \cap J^{l}(p) \neq \emptyset$. Assume first that $J^{l}(\bar{p}) \cap J^{l}(p)=\emptyset$. Take $j \in J^{l}(\bar{p})$, then there is an $u>l$ such that $j \in$ $J^{u}(p)$. Because of Lemma $4.1 \theta\left(\hat{p} A f_{j}\right) \succ_{L} \theta\left(\bar{p} A f_{j}\right)=a^{l}(\bar{p})$. Let $j \in J^{u}(\bar{p})$, with $u>l$. Then $\theta\left(\bar{p} A f_{j}\right) \succ_{L} a^{l}(\bar{p})$ and $\theta\left(p A f_{j}\right) \succeq_{L} a^{l}(\bar{p})$. Applying Lemma 4.1 gives $\theta\left(\hat{p} A f_{k}\right) \succ_{L} a^{l}(\bar{p})$. We can conclude that

$$
\theta\left(\hat{p} A f_{j}\right) \succ_{L} a^{l}(\bar{p}),
$$

for all $j \in\{1, \ldots, n\} \backslash \bigcup_{k=1}^{l-1} J^{k}(p)$. Hence, $a^{l}(\hat{p}) \succ_{L} a^{l}(\bar{p})$ and $\hat{p} \succ_{\text {prot }} \bar{p}$.

Now assume $J^{l}(\bar{p}) \cap J^{l}(p) \neq \varnothing$. Then there is also a level of $\hat{p}$ with the same vector value, since for all $j \in J^{l}(\bar{p}) \cap J^{l}(p) \neq \emptyset$, it holds that $\theta\left(\hat{p} A f_{j}\right)=\theta\left(\bar{p} A f_{j}\right)=$ $\theta\left(p A f_{j}\right)=a^{l}(\bar{p})$. As we have seen before it follows from Lemma 4.1 that for all $j \notin \bigcup_{t=1}^{l}\left(J^{t}(\bar{p}) \cap J^{t}(p)\right)$ :

$$
\theta\left(\hat{p} A f_{j}\right) \succ_{L} a^{l}(\bar{p}) .
$$

Hence $a^{l}(\hat{p})=a^{l}(\bar{p})$ and $J^{l}(\hat{p}) \varsubsetneqq J^{l}(\bar{p})$. So $\hat{p} \succ_{\text {prot }} \bar{p}$, establishing a contradiction.

Definition 4.2 Let $\Gamma=\left\langle\Delta_{m}, \Delta_{n}, A, B\right\rangle$ be a multicriteria game. Let $p, \bar{p} \in \Delta_{m}$. We say that $\bar{p}$ prudently dominates $p$, in notation, $\bar{p} \succ_{\text {pru }} p$, if there exists an $l \in \mathbb{N}$ such that

(i) $a_{1}^{t}(\bar{p})=a_{1}^{t}(p)$ and $\left|J^{t}(\bar{p})\right|=\left|J^{t}(p)\right|$ for all $t \in \mathbb{N}, t<l$,

(ii) $a_{1}^{l}(\bar{p}) \succ_{L} a_{1}^{l}(\sigma)$ or both $a_{1}^{l}(\bar{\sigma})=a_{1}^{l}(\sigma)$ and $\left|J^{l}(\bar{p})\right|<\left|J^{l}(p)\right|$. 
A mixed strategy $p \in \Delta_{m}$ is called prudent for player 1 in $\Gamma$ if there does not exist a mixed strategy $\bar{p} \in \Delta_{m}$ such that $\bar{p} \succ_{\text {pru }} p$.

Notice that any prudent strategy is also protective, since if a strategy is protectively dominated, it is also prudently dominated by the same strategy. The following lemma proves the converse with the use of Lemma 4.2.

Lemma 4.3 In any multicriteria game, a mixed strategy for player 1 is protective if and only if it is prudent.

Proof Let $\Gamma=\left\langle\Delta_{m}, \Delta_{n}, A, B\right\rangle$ be a multicriteria game. It remains to prove that any protective strategy is also prudent. Let $p \in \Delta_{m}$ be a protective strategy and suppose $p$ is not a prudent strategy. Then, by definition, there exists a strategy $\bar{p}$ such that $\bar{p} \succ_{\text {pru }} p$. This means that $p$ and $\bar{p}$ are not payoff equivalent. By Lemma 4.2, $p \succ_{\text {pro }}$ $\bar{p}$, from which it follows that $p \succ_{p r u} \bar{p}$. This is a contradiction.

The following theorem guarantees the existence of a prudent (protective) strategy combination in any two-person multicriteria game.

Theorem 4.1 Every multicriteria two-person game has at least one prudent strategy combination.

Proof It suffices to prove the existence of a prudent strategy for player 1 . Let $\Gamma=$ $\left\langle\Delta_{m}, \Delta_{n}, A, B\right\rangle$ be a multicriteria game. Define the sets

$$
\begin{aligned}
& M^{1}=\left\{p \in \Delta_{m} \mid a^{1}(p) \succeq_{L} a^{1}(\bar{p}) \text { for every } \bar{p} \in \Delta_{m}\right\}, \\
& P^{1}=\left\{p \in M^{1}|| J^{1}(p)|\leq| J^{1}(\bar{p}) \mid \text { for any } \bar{p} \in M^{1}\right\},
\end{aligned}
$$

and for every $t>1$,

$$
\begin{aligned}
& M^{t}=\left\{p \in P^{t-1} \mid a^{t}(p) \succeq_{L} a^{t}(\bar{p}) \text { for every } \bar{p} \in P^{t-1}\right\}, \\
& P^{t}=\left\{p \in M^{t}|| J^{t}(p)|\leq| J^{t}(\bar{p}) \mid \text { for any } \bar{p} \in M^{t}\right\} .
\end{aligned}
$$

It follows from the continuity of the function $\theta$ and the compactness of $\Delta_{m}$ that $M^{1}$ and $P^{1}$ are nonempty compact sets. ${ }^{3}$ It follows by induction that $M^{t} \neq \varnothing, P^{t} \neq \varnothing$ and compact for any $t \in \mathbb{N}$. Moreover, for every $p, \bar{p} \in M^{t}$ we have $a_{1}^{t}(p)=a_{1}^{t}(\bar{p})$ and for every $p, \bar{p} \in P^{t}$ it holds that $a^{l}(p)=a^{l}(\bar{p})$ and $\left|J^{l}(\bar{p})\right|=\left|J^{l}(p)\right|$, for all $l \in\{1, \ldots, t\}$. Since player 2 has finitely many pure strategies, there exists a $s \in \mathbb{N}$ such that for all $p \in P^{s}: \bigcup_{l=1}^{s} J^{l}(p)=\{1, \ldots, n\}$ and $\left|J^{s}(p)\right|>0$. Note that for all $l>s, P^{l}=P^{s}, a^{l}(p)=(\infty, \ldots, \infty)$ and $J^{l}(p)=\emptyset$ for all $p \in P^{l}$. By definition the set $P^{s}$ is precisely the set of prudent strategies for player 1 .

In the following two examples the notion of protective strategy combinations is illustrated in a zero-sum like environment. In the first example it is shown that in a

\footnotetext{
${ }^{3}$ In fact $P^{1}$ is a finite union of finite intersections of inverse images of compact sets, hence $P^{1}$ is compact itself.
} 
game with a large set of equilibria protectiveness picks the intuitively logic strategic combination. In the second example it is shown that a protective strategy combination need not necessarily be a (Pareto) equilibrium.

Example 4.1 Consider the two-person multicriteria game $\left(\Delta_{2}, \Delta_{2}, A, B\right)$, where $B=$ $-A$ and

$$
A=\left(\begin{array}{ll}
(0,1) & (1,0) \\
(1,0) & (0,1)
\end{array}\right)
$$

The set of Pareto equilibria equals $\Delta_{2} \times \Delta_{2}$, so each strategy combination is an equilibrium. Let $\bar{p}=(p, 1-p)$, with $p \in[0,1]$ be a strategy of player one. Then $\theta\left(\bar{p} A f_{1}\right)=\theta\left(\bar{p} A f_{2}\right)=(p, 1-p)$ if $p \leq \frac{1}{2}$ and $\theta\left(\bar{p} A f_{1}\right)=\theta\left(\bar{p} A f_{2}\right)=(1-p, p)$ if $p \geq \frac{1}{2}$. Hence for player one, only the strategy $\left(\frac{1}{2}, \frac{1}{2}\right)$ is protective. Obviously, also $\left(\frac{1}{2}, \frac{1}{2}\right)$ is the only protective strategy of player 2 .

Example 4.2 Consider the two-person multicriteria game $\left(\Delta_{2}, \Delta_{2}, A, B\right)$, where

$$
A=\left(\begin{array}{ll}
(0,0) & (3,-2) \\
(1,1) & (5,-3)
\end{array}\right) \quad \text { and } \quad B=\left(\begin{array}{cc}
0 & -3 \\
-1 & -5
\end{array}\right) \text {. }
$$

Clearly, the only protective strategy of player one is $e_{1}$. the only protective strategy of player two is $f_{1}$. The unique protective strategy combination $\left(e_{1}, f_{1}\right)$ is not an equilibrium, since player one can deviate to $e_{2}$, achieving a higher payoff in both criteria.

Open Access This article is distributed under the terms of the Creative Commons Attribution Noncommercial License which permits any noncommercial use, distribution, and reproduction in any medium, provided the original author(s) and source are credited.

\section{References}

1. Blackwell, D.: An analog of the minimax theorem for vector payoffs. Pac. J. Math. 6, 1-8 (1956)

2. Shapley, L.: Equilibrium points in games with vector payoffs. Nav. Res. Logist. Q. 1, 57-61 (1959)

3. Nash, J.: Noncooperative games. Ann. Math. 54, 575-625 (1951)

4. Borm, P., van den Aarssen, J., Tijs, S.: Pareto equilibria in multiobjective games. Methods Oper. Res. 60, 303-312 (1988)

5. Zhao, J.: The equilibria of a multiple objective game. Int. J. Game Theory 20, 171-182 (1991)

6. Geoffrion, A.: Proper efficiency and the theory of vector maximization. J. Math. Anal. Appl. 22, 618-630 (1968)

7. Selten, R.: Reexamination of the perfectness concept for equilibrium points in extensive games. Int. J. Game Theory 4, 25-55 (1975)

8. Borm, P., van Megen, F., Tijs, S.: A perfectness concept for multicriteria games. Math. Methods Oper. Res. 49, 401-412 (1999)

9. Myerson, R.: Refinements of the Nash equilibrium concept. Int. J. Game Theory 7, 73-80 (1978)

10. Fiestras-Janeiro, G., Borm, P., van Megen, F.: Protective and prudent behavior in games. J. Econ. Theory 78, 167-175 (1998) 